\title{
Análise microbiológica da água fornecida a uma Unidade de Saúde no Município de
}

\section{Itajubá, Minas Gerais}

\author{
Microbiological analysis of the water provided to a Health Unit in the Municipality of Itajubá, \\ Minas Gerais \\ Análisis microbiológico del agua suministrada a una Unidad de Salud del Municipio de Itajubá, \\ Minas Gerais
}

Ana Carolina França ORCID: https://orcid.org/0000-00002-6594-5027 Centro Universitário de Itajubá, Brasil

E-mail: anacarolinadfranca22@gmail.com

Guilherme Passos de Abreu

ORCID: https://orcid.org/0000-0001-9303-9999 Centro Universitário de Itajubá, Brasil E-mail: guipassosabreu@hotmail.com

Laiz Furlan Balioni

ORCID: https://orcid.org/0000-0002-7744-3948 Centro Universitário de Itajubá, Brasil E-mail: laizfurlan@gmail.com

Patrícia Capellato

ORCID: https://orcid.org/0000-0002-6397-5820 Universidade Federal de Itajubá, Brasil E-mail: pcapellato@gmail.com

Maria Gabriela Araújo Ranieri ORCID: https://orcid.org/0000-0001-8631-020X Universidade Federal de Itajubá, Brasil E-mail: gabiranieri@unifei.edu.br

Ana Lúcia Fonseca

ORCID: https://orcid.org/0000-0003-4339-6503 Universidade Federal de Itajubá, Brasil E-mail: afonseca@unifei.edu.br

Gilza Carla Ribeiro

ORCID: https://orcid.org/0000-0002-1724-893X Universidade Federal de Itajubá, Brasil E-mail: gilza.carla@zipmail.com.br

Paulo Sergio Marques

ORCID: https://orcid.org/0000-0003-0330-0933 Universidade Federal de Itajubá, Brasil E-mail: marquespaulo@unifei.edu.br

Daniela Sachs

ORCID: https://orcid.org/0000-0002-3767-2455 Universidade Federal de Itajubá, Brasil E-mail: danisachs@unifei.edu.br

\section{Resumo}

O controle de qualidade da água é uma das ações fundamentais para a prevenção de doenças. Uma das causas mais frequentes de morte no mundo são as doenças transmitidas pela água, onde as bactérias contaminantes mais encontradas são Acinetobacter spp, Escherichia coli, Klebsiella pneumoniae, Pseudomonas aeruginosa e Staphylococcus aureus. A falta da potabilidade da água nem sempre é perceptível à visão ou olfato, sendo necessária uma análise laboratorial para detectá-la. Como a qualidade da água é um fator imprescindível à manutenção da saúde humana, em ambientes de saúde, faz-se necessária a confirmação da qualidade da água oferecida aos pacientes. Assim, foi selecionado o ponto de coleta de amostras de água em uma Unidade de Saúde localizados no município de Itajubá, Minas Gerais. Foi realizada a análise de presença de coliformes totais e de Escherichia coli por utilizando o reagente Colilert®, que é aprovado pelo método padrão para análise de água e esgoto Standard Methods for the Examination of Water and Wastewater. Os resultados foram avaliados por alteração de cor indicativa de presença ou ausência de coliformes totais e E. coli. Na Unidade de Saúde, todas as amostras, foram negativas para coliformes totais e presença de E.coli, indicando que as amostras são próprias para consumo. O objetivo desse trabalho foi verificar a qualidade microbiológica da água que é fornecida a uma Unidade de Saúde e alertar para a importância do 
monitoramento da qualidade visando a prevenção e disseminação de doenças que podem agravar ainda mais o quadro de pacientes já debilitados.

Palavras-chave: Qualidade da água; Coliformes fecais; Saúde pública.

\begin{abstract}
Water quality control is one of the fundamental actions for disease prevention. One of the most frequent causes of death in the world is water-borne diseases, where the most commonly found contaminating bacteria are Acinetobacter spp, Escherichia coli, Klebsiella pneumoniae, Pseudomonas aeruginosa and Staphylococcus aureus. The lack of potable water is not always noticeable to sight or smell, requiring a laboratory analysis to detect it. As water quality is an essential factor in maintaining human health, in health settings, it is necessary to confirm the quality of the water offered to patients. Thus, the point of collection of water samples was selected in a Health Unit located in the municipality of Itajubá, Minas Gerais. The analysis of the presence of total coliforms and Escherichia coli was performed using the Colilert ${ }^{\circledR}$ reagent, which is approved by the standard method for water and sewage analysis Standard Methods for the Examination of Water and Wastewater. The results were evaluated by changing the color indicating the presence or absence of total coliforms and E. coli. At the Health Unit, all samples were negative for total coliforms and the presence of E. coli, indicating that the samples are suitable for consumption. The objective of this work was to verify the microbiological quality of the water that is provided to a Health Unit and to warn of the importance of quality monitoring aiming at the prevention and dissemination of diseases that can further aggravate the situation of already debilitated patients.
\end{abstract}

Keywords: Water quality; Fecal coliforms; Public health.

\title{
Resumen
}

El control de la calidad del agua es una de las acciones fundamentales para la prevención de enfermedades. Una de las causas de muerte más frecuentes en el mundo son las enfermedades transmitidas por el agua, donde las bacterias contaminantes más comúnmente encontradas son Acinetobacter spp, Escherichia coli, Klebsiella pneumoniae, Pseudomonas aeruginosa y Staphylococcus aureus. La falta de agua potable no siempre es perceptible a la vista $u$ olfato, requiriendo un análisis de laboratorio para detectarla. Dado que la calidad del agua es un factor esencial para el mantenimiento de la salud humana, en los entornos sanitarios es necesario confirmar la calidad del agua que se ofrece a los pacientes. Así, se seleccionó el punto de recolección de muestras de agua en una Unidad de Salud ubicada en el municipio de Itajubá, Minas Gerais. El análisis de la presencia de coliformes totales y Escherichia coli se llevó a cabo utilizando el reactivo Colilert®, que está aprobado por el método estándar para análisis de agua y aguas residuales Métodos estándar para el examen de agua y aguas residuales. Los resultados se evaluaron cambiando el color que indica la presencia o ausencia de coliformes totales y E. coli. En la Unidad de Salud, todas las muestras resultaron negativas para coliformes totales y la presencia de E. coli, lo que indica que las muestras son aptas para el consumo. El objetivo de este trabajo fue verificar la calidad microbiológica del agua que se suministra a una Unidad de Salud y advertir de la importancia del monitoreo de la calidad con el objetivo de prevenir y diseminar enfermedades que pueden agravar aún más la situación de los pacientes ya debilitados.

Palabras clave: Calidad del agua; Coliformes fecales; Salud pública.

\section{Introdução}

A água é primordial para a manutenção da vida do homem e para a estabilidade de toda a natureza. A sua importância torna se hoje uma preocupação mundial frente aos riscos de poluição, do consumo desordenado, das variações climáticas e no uso desordenado do solo, além dos riscos de ausência hídrica. Além disso, a água tem influência na qualidade de vida da população carente como um veículo capaz de transmitir uma série de agentes patogênicos (Nogueira et al., 2018).

A saúde da população está relacionada a qualidade da água a qual a mesma está exposta. Os principais contaminantes presentes na água são os coliformes totais e termotolerantes. Estes podem ser utilizados como indicadores do contato direto ou indireto com material fecal (Porcy et al., 2019). As bactérias são responsáveis por aproximadamente $90 \%$ dos casos infeções hídricas e/ou intoxicações alimentares, e as mais encontradas são Escherichia coli, Salmonella spp., Staphylococcus aureus e Yersinia enterocolitica(Sales, Tunala, Vasco, Ravazzani, \& Caveião, 2015).

Desta forma, é necessário que as pessoas tenham acesso a água de qualidade, do ponto de vista químico e microbiológico e isso só é possível através de tratamentos. Quando o primeiro Índice de Qualidade da Água (IQA) foi desenvolvido por Horton em 1960 muitas metodologias de análise da qualidade da água foram propostos (Tian \& Wu, 2019). O rápido desenvolvimento de metodologias, aumenta a compreensão da qualidade da água por uma simples leitura visual ou 
avaliação numérica, ou por uma interpretação mais complexa das características da qualidade da água ( $\mathrm{Su}, \mathrm{Wu}$, \& $\mathrm{He}, 2019)$.

O IQA (Índice de Qualidade da Água) em Minas Gerais de acordo com (Amâncio, Coelho, Marques, Viola, \& De Mello, 2018) foi classificado como ruim em todos os pontos de monitoramento, devido às atividades entorno da bacia, como a pecuária, irrigação e as poluições pontuais. Dependendo dos contaminantes presentes, diferentes tecnologias e suas combinações podem ser usadas para a produção de água potável (de Moel, Verberk, \& van Dijk, 2006).

Entre o ponto de saída da ETA e a chegada à torneira do usuário, a água potável passa pela rede de distribuição, onde fica por períodos que variam normalmente de horas a dias. Durante esse tempo a agua pode sofrer várias contaminações por meio de vários processos, entre eles, a formação de biofilme (Manuel, Nunes, \& Melo, 2007) apresentando riscos potencialmente graves para a saúde dos consumidores.

A Organização Mundial da Saúde estima que cerca de 2100 milhões de pessoas não terão acesso a água potável, em 2030 e que atualmente, cerca de 844 milhões pessoas carecem de serviço básico de água potável e 159 milhões de pessoas ainda estão bebendo água não tratada de fontes como riachos, lagos e reservas (WHO Joint Expert Meeting, 2013) A carência de água potável é responsável por $85 \%$ das doenças e mortes no mundo todo. Na América Latina, cerca de 150.000 mortes ocorrem por doenças associadas a água não potável, e 85\% envolvem crianças menores de 5 anos. No equador, apenas $70 \%$ da população tem água potável, e os outros $30 \%$ consomem água diariamente com contaminação de fezes.

Por isso, esse trabalho apresenta um carater social e tem como objetivo verificar a qualidade microbiológica da água que é fornecida a uma Unidade de Saúde com o intuito de concientizar sobre a importância do monitoramento da qualidade visando a prevenção e disseminação de doenças que podem agravar ainda mais o quadro de pacientes já debilitados. Foi empregadauma metodologia qualitativa. A partir desses aspectos, algumas metodologias de observações, mapeamento da área e análises foram adotadas, pois Segundo Shitsuka 2018, o emprego de um método facilita o caminho de uma descoberta (Pereira, Shitsuka, Parreira, \& Shitsuka, 2018).

\section{Metodologia}

Estudo empregando método qualitativo valoriza a interpretação dos resultados por parte do pesquisador com suas opiniões sobre o fenômeno em estudo assim como os dados comprobatórios (Lüdke \& André, 1986). Assim, foram coletadas amostras de água em três pontos diferentes de uma Unidade de Saúde no Município de Itajubá, localizado ao Sul do Estado de Minas Gerais. Em cada ambiente da Unidade de Saúde, foi realizada a coleta das amostras de água em triplicata que foram acondicionadas em recipientes de plástico estéril. Para tanto, as torneiras foram abertas por 40 segundos e após esse tempo foi feita a captação das amostras. Foram coletados $30 \mathrm{~mL}$ de amostra de água corrente para cada amostra. Devido a importância da metodologia para a confiabilidade dos resultados (Estrela 2018) e Koche 2011, o transporte das amostras até ao Laboratório de Microbiologia Aplicada da Unifei foi efetuado em mala térmica imediatamente após a coleta. Para a análise qualitativa de coliformes totais e Escherichia coli (E. coli) nas amostras de água foi escolhido o método do substrato cromogênico e fluorogênico, COLILERT® (IDEXX). Este método se baseia na identificação dos microrganismos pela análise de suas enzimas constituintes (Ramoutar, 2020) (Covert et al., 1989, Silva et al., 2017) aprovado pelo Standard Methods for the Examination of Water and Wastewater (Rodger B. Baird, 2017) Para tanto, em câmara de fluxo laminar, higienizada com etanol a 70\% e após incidência de luz ultravioleta (UV) por 15 minutos, adicionou-se a cada $30 \mathrm{~mL}$ de amostra de água, o reagente de Colilert ${ }^{\circledR}$ e foi feita a homogeneização. As amostras foram mantidas em estufa à $37^{\circ} \mathrm{C}$ por $24 \mathrm{~h}$. Após este período, realizou-se a leitura de coloração por observação macroscópica de alteração da coloração do meio (de incolor para amarelo se positivo para coliformes totais) e de fluorescência (fluorescente se positivo para E. coli), com o auxílio de lâmpada UV a $365 \mathrm{~nm}$. No método cromogênico e fluorogênico COLILERT® estão presentes o substrato orto-nitrofenil- $\beta$-Dgalactopiranosídeo (ONPG) que é cromogênico e o substrato fluorogênico 4-metilumbeliferil- $\beta$-D-glucoronídeo (MUG) que é 
fluorogênico e que indicam a presença de bactérias do grupo coliforme total e E. coli em amostras de água, respectivamente (Manafi, 2000). O substrato cromogênico ONPG é usado na deteç̧ão da enzima típica do grupo coliformes totais, a $\beta$ galactosidase, que hidrolisa o ONPG a orto-nitrofenol resultando na alteração da cor do meio de incolor para amarelo (Hunt; Rice, 2005; Silva et al., 2005, Silva et al., 2017). A E. coli por sua vez utiliza $\square$-glucuronidase para metabolizar o MUG que é degradado na presença da enzima $\beta$-glicuronidase produzida pela Escherichia coli resultando em 4-metilumbeliferona que possui uma fluorescência observada sob lâmpada ultravioleta a 365nm (Covert et al., 1989; Silva et al., 2005, Silva et al., 2017).

Se ocorrer alteração de cor de incolor para amarelo e não apresentar fluorescência sob luz UV, indica presença de bactérias do grupo coliforme e ausência de E. coli na amostra. Caso a cor do meio seja alterada para amarela e este apresentar fluorescência sob luz UV, significa que bactérias do grupo coliforme e E. coli estão presentes na amostra analisada.

\section{Resultados e Discussão}

O combate a microrganismos patogênicos que possam vir a causar surtos é o principal foco no tratamento da água antes da distribuição para a população, a qualidade da água é uma das ações fundamentais para a prevenção de doenças principalmente em crianças (Khan, Wojdyla, Say, Gülmezoglu, \& Van Look, 2006). A pesquisa de microrganismos na água é necessária uma vez que há microrganismos, que são parte daquele ambiente (Gameiro et al., 2020). A análise qualitativa (Mushavi et al., 2020), quanto a presença de coliformes totais, da água coletada em três pontos distintos da unidade de saúde, indicou, macroscopicamente, que, apenas uma das amostras (indicada pelo número 2.1, Figura 1) apresentou coloração levemente amarelada. Contudo, apesar da leve alteração de cor, a comparação com padrão fornecido pela empresa, fabricante do teste qualitativo, indica que não houve alteração significativa, levando a concluir que não há presença de coliformes totais nas amostras de água coletadas em todos os pontos da Unidade de Saúde, o que indica que todas as amostras são próprias ao consumo humano e uso nas tarefas de rotina.

Figura 1. Amostras de água da Unidade de saúde do Município de Itajubá, MG, para teste macroscópico qualitativo para detecção de coliformes fecais e $E$. coli.

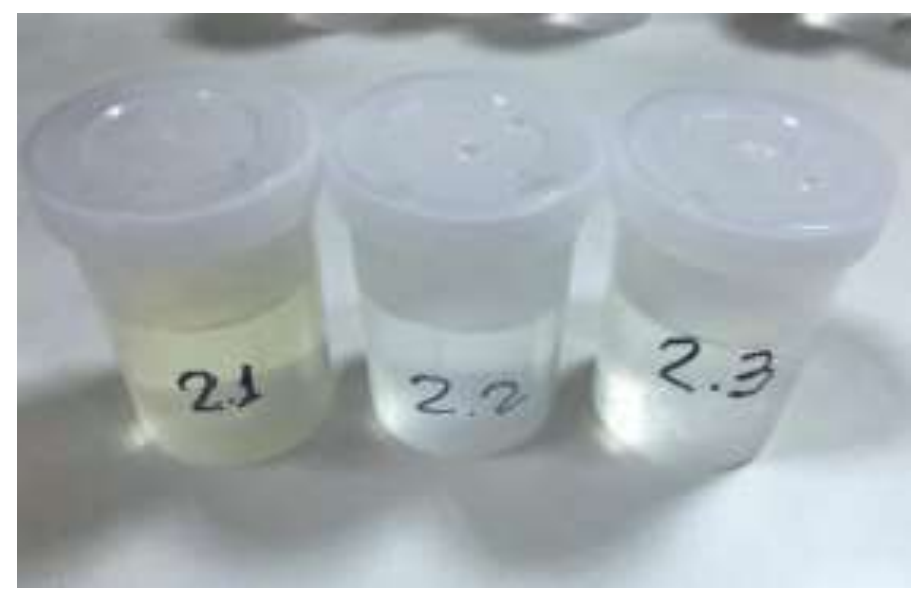

Fonte: Autores.

Apesar da alteração leve na cor, a comparação com o padrão fornecido pela empresa, permite concluir que a presença de coliformes totais é muito baixa, não sendo suficiente para desqualificar a amostra que, portanto, apresenta-se própria ao consumo humano $(2914 / 2011,2011)$. O Colilertß (IDEXX) foi concebido para a análise simultânea de coliformes e E. coli em amostras de águas e tem sido bastante usado em análise de rotina de águas potáveis, de piscinas, doces, costeiras e até 
residuais.

O tratamento da água é garantido por lei e deve estar de acordo com a portaria n ${ }^{\circ} 2.914$, do Ministério da Saúde, 12 de dezembro de 2011 que regulamenta as características físico-químicas e microbiológicas, que uma água potável deve possuir. Esta portaria, permite a presença de bactérias mesófilas, isto é, bactérias que se multiplicam na faixa de 20 a $40^{\circ} \mathrm{C}$ de temperatura (Van Haute et al., 2020). Nos limites aceitáveis, esses tipos de microrganismos não são prejudiciais à saúde, mas devem ser constantemente monitorados, uma vez que seu aumento pode trazer algum dano à população (Portaria MS, 2012).

Há a necessidade de cuidados no abastecimento de hospitais e clínicas, onde há pacientes imunodeprimidos, pois são pacientes mais expostos a contaminação. O sistema imunodeprimido torna os pacientes susceptíveis à infecções. Uma das medidas profiláticas é evitar a exposição a fontes de água de origem duvidosa, uma vez que organismos menos virulentos progressivamente se tornam mais perigosos. O quadro geral da imunossupressão do paciente, que determina seu risco para a infecção, é afetado por várias variáveis: idade, dose e duração de terapia imunossupressora, estado da defesa humoral, integridade da pele e mucosas, fatores metabólicos, presença ou ausência de infecções imunomoduladoras, como HIV, citomegalovírus, vírus da Hepatite e vírus Epstein-Barr (Slavish, 2012). Essas infecções constituem importante causa de morbidade e mortalidade nos hospitais, ocasionando aumento nos gastos dos cofres públicos. Um estudo mostra que cerca de 100 mil pessoas por ano morrem vítimas destas infecções no Brasil (Barros et al., 2015). O Ministério da Saúde, regulamenta a qualidade do sistema de abastecimento de águas com base na presença de coliformes e contagem de bactérias heterotróficas, enquanto a regulamentação mais restritiva, existe em relação à água mineral natural e proíbe a presença de bactérias potencialmente patogênicas, incluindo Pseudomonas aeruginosa, Enterococcus spp. e Clostridium perfringens. Todas as amostras analisadas foram negativas para a presença de E.coli, como ilustrado na Figura 2. Na imagem, as amostras são comparadas a um frasco contendo amostra positiva para E.coli, que apresenta emissão de fluorescência. Em nenhuma das amostras coletadas, houve emissão de fluorescência, indicativa da presença da bactéria.

Figura 2. Análise qualitativa das amostras de água de uma unidade de saúde de Itajubá-MG, por fluorescência.

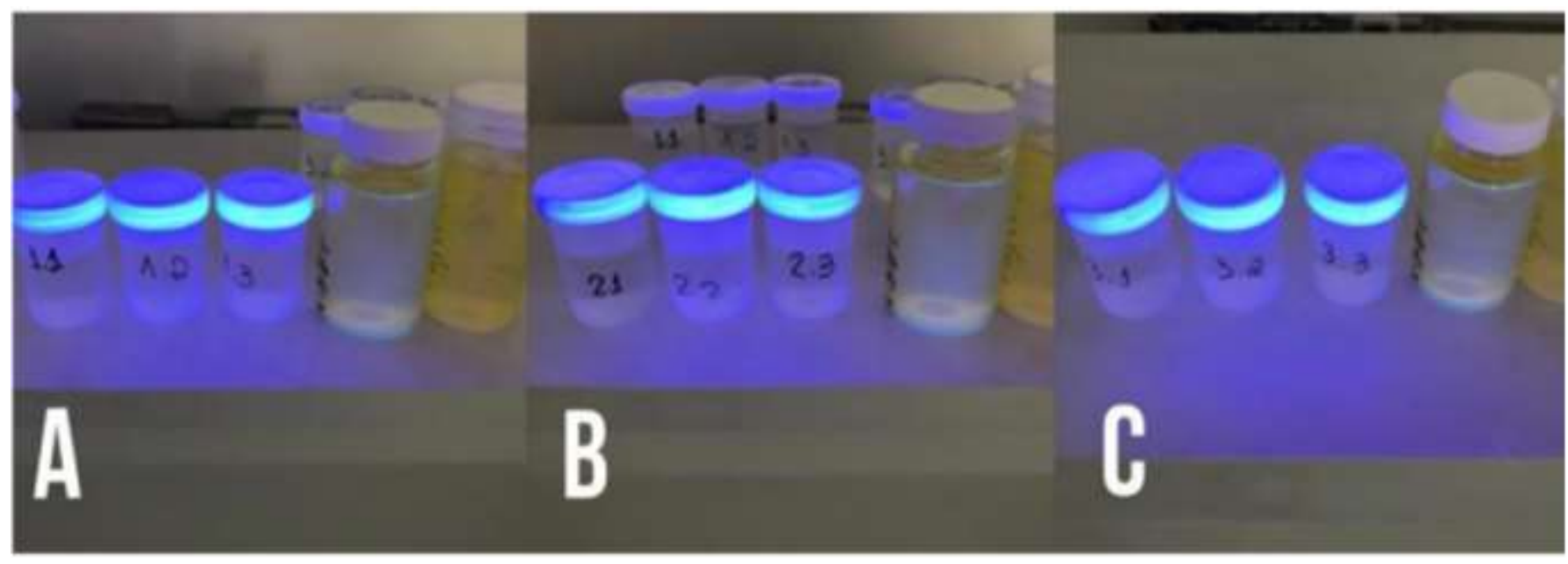

Fonte: Autores.

Os coliformes totais e coliformes termotolerantes, são bactérias que sobrevivem tanto na presença como na ausência de oxigênio (aeróbicas e anaeróbicas facultativas), são bacilos Gram-negativos, não esporulados e são encontradas frequentemente na microbiota intestinal do ser humano e de animais, sendo, portanto, a Escherichia coli, a principal indicadora de contaminação fecal humana (Brasil, 2013). A análise qualitativa para coliformes fecais, está apresentada na tabela 1. Todos os três pontos de coleta das amostras na Unidade de Saúde, apresentaram-se negativas para coliformes totais. Este resultado 
comprova experimentalmente, que a 25 água oferecida aos pacientes da unidade de saúde objeto do presente estudo, está em boas condições para o consumo e uso em atividades rotineiras, livre de coliformes totais e termotolerantes ( $\mathrm{Zhao,} \mathrm{Li}$, Chen, Meng, \& Zheng, 2020).

Tabela 1. Análise qualitativa de coliformes totais e E.coli, presentes na água de uma unidade de saúde no município de Itajubá-MG.

\begin{tabular}{ccl}
\hline Amostra Unidade de Saúde & Coliformes totais & $\boldsymbol{E . c o l i}$ \\
\hline 1.1 & Negativo & Negativo \\
\hline 1.2 & Negativo & Negativo \\
\hline 1.3 & Negativo & Negativo \\
\hline 2.1 & Negativo & Negativo \\
\hline 2.2 & Negativo & Negativo \\
\hline 2.3 & Negativo & Negativo \\
\hline 3.1 & Negativo & Negativo \\
\hline 3.2 & Negativo & Negativo \\
\hline 3.3 & Negativo & Negativo \\
\hline
\end{tabular}

Fonte: Autores.

\section{Conclusão}

O método Colilert® (IDEXX), demonstrou ser de fácil uso, rápido e preciso para as análises propostas. Os resultados obtidos demonstram que a água tratada disponibilizada na Unidade de Saúde está de acordo com os padrões de potabilidade, uma vez que todos os pontos de coleta das amostras apresentaram-se isentos de coliformes totais e Escherichia coli, estando, portanto, em qualidade adequada para consumo e fornecimento aos pacientes. É necessária atenção quanto aos padrões de potabilidade da água oferecida principalmente à pacientes em estado de vulnerabilidade, pois à água é veículo de transmissão de doenças que podem agravar ainda mais o estado de saúde de pacientes. No futuro pretendemos investigar a qualidade da água em outras Unidades básicas de Saúde, assim como alguns hospitais da região visto a importância da investigação da qualidade da água para a população.

\section{Referências}

Nogueira, E.F.R., Silva, A.R.L., Miranda Junior, R.N.C, Moura, L.B.D., Cavalcante, L.G.C.C., Leitão, J.M.S.R., Feerreira,

V.S., Carvalho, C.I.M., Sousa, S.B.G. (2018). Análise comparativa da qualidade da água disponível para consumo nos bebedouros de escolas públicas do município de Santo Inácio do Piauí. Revista Eletrônica Acervo Saúde, 14, S1742-S1746

Amâncio, D. V., Coelho, G., Marques, R. F. de P. V., Viola, M. R., \& De Mello, C. R. (2018). Qualidade Da Água Nas Sub-Bacias Hidrográficas Dos Rios Capivari E Mortes, Minas Gerais. Scientia Agraria. https://doi.org/10.5380/rsa.v19i1.53175

de Moel, P. J., Verberk, J. Q. J. C., \& van Dijk, J. C. (2006). Drinking water: Principles and practices. Drinking Water: Principles and Practices. World Scientific Publishing Co. https://doi.org/10.1142/6135

Gameiro, E., Bugno, A., Pontes De Lima, F., I, S., Aparecida, A., Almodovar, B., \& Paulo, S. (2020). Avaliação da técnica de detecção por fluorescência como alternativa para contagem de bactérias heterotróficas em água para hemodiálise Evaluation of fluorescence detection technique as an alternative for heterotrophic bacteria count in hemodialysis water. Vigilância Sanitária Em Debate: Sociedade, Ciência \& Tecnologia (Health Surveillance under Debate: Society, Science \& Technology) - Visa Em Debate, 8(2), 94-105. https://doi.org/10.22239/2317-269x.01387

Khan, K. S., Wojdyla, D., Say, L., Gülmezoglu, A. M., \& Van Look, P. F. (2006). WHO analysis of causes of maternal death: a systematic review. Lancet, 367(9516), 1066-1074. https://doi.org/10.1016/S0140-6736(06)68397-9

Lüdke, M., \& André, M. E. D. A. (1986). Pesquisa em Educação: Abordagens Qualitativas. Temas Básicos de Educação e Ensino.

Manuel, C. M., Nunes, O. C., \& Melo, L. F. (2007). Dynamics of drinking water biofilm in flow/non-flow conditions. Water Research, 41(3), 551-562. 
Research, Society and Development, v. 10, n. 6, e33910615220, 2021

(CC BY 4.0) | ISSN 2525-3409 | DOI: http://dx.doi.org/10.33448/rsd-v10i6.15220

https://doi.org/10.1016/j.watres.2006.11.007

Mushavi, R. C., Burns, B. F. O., Kakuhikire, B., Owembabazi, M., Vořechovská, D., McDonough, A. Q., \& Tsai, A. C. (2020). "When you have no water, it means you have no peace": A mixed-methods, whole-population study of water insecurity and depression in rural Uganda. Social Science and Medicine, 245, 112561. https://doi.org/10.1016/j.socscimed.2019.112561

Pereira, A. S., Shitsuka, D. M., Parreira, F. J., \& Shitsuka, R. (2018). Metodologia da Pesquisa Científica Locais da Ciência Metodologia da Pesquisa Científica Metodologia da Pesquisa Científica. UFSM.

Porcy, C., Costa, S., Helena, V., Monteiro, S., Nogueira, J. N., Alex, R., \& Menezes, D. O. (2019). Avaliação microbiológica da água de consumo de casas localizadas em área alagada em um município do estado Amapá. Revista Eletrônica Acervo Saúde / Electronic Journal Collection Health, 12(4), 1-9.

Ramoutar, S. (2020). The use of Colilert-18, Colilert and Enterolert for the detection of faecal coliform, Escherichia coli and Enterococci in tropical marine waters, Trinidad and Tobago. Regional Studies in Marine Science, 40, 101490. https://doi.org/10.1016/j.rsma.2020.101490

Rodger B. Baird, C. E. W. R. A. D. E. (2017). Standard Methods for the Examination of Water and Wastewater, (23a ed.),

Sales, W. B., Tunala, J. F., Vasco, J. F. de M., Ravazzani, E. D. do A., \& Caveião, C. (2015). Occurrence of Total and Thermotolerant Coliforms in fried pastries sold in bars in downtown Curitiba-PR, Brazil. Demetra: Food, Nutrition \& Health, 10(1), 77.

Su, F., Wu, J., \& He, S. (2019). Set pair analysis-Markov chain model for groundwater quality assessment and prediction: A case study of Xi'an city, China. Human and Ecological Risk Assessment, 25(1-2), 158-175. https://doi.org/10.1080/10807039.2019.1568860

Tian, R., \& Wu, J. (2019). Groundwater quality appraisal by improved set pair analysis with game theory weightage and health risk estimation of contaminants for Xuecha drinking water source in a loess area in Northwest China. Human and Ecological Risk Assessment, 25(1-2), 132-157. https://doi.org/10.1080/10807039.2019.1573035

Van Haute, S., Luo, Y., Bolten, S., Gu, G., Nou, X., \& Millner, P. (2020). Survival of Salmonella enterica and shifts in the culturable mesophilic aerobic bacterial community as impacted by tomato wash water particulate size and chlorine treatment. Food Microbiology, 90, 103470. https://doi.org/10.1016/j.fm.2020.103470

WHO Joint Expert Meeting. (2013). World Health Organization Water Quality and Health Joint Expert Meeting Organized in coordination with, (March). 